e-Journal of Educational

Research, Assessment and Evaluation

\section{REIIEVE}

Revista ELectrónica de Investigación y EValuación Educativa

\title{
LAS REVISTAS MEXICANAS DE INVESTIGACIÓN EDUCATIVA RUMBO A LA CORRIENTE PRINCIPAL DE DIFUSIÓN DE LA CIENCIA
}

\section{[Mexican journals of educational research towards mainstream dissemination of science]}

by/por

\begin{abstract}
Article record
About authors

HTML format
\end{abstract}

\author{
Cabrera-Flores, Mayer \\ Luna-Serrano, Edna (eluna@uabc.edu.mx) \\ Vidauri, Gabriela
}

Ficha del artículo

$\underline{\text { Sobre los autores }}$

Formato HTML

\begin{abstract}
The purpose of this study is to analyze the Electronic Journal of Educational Research (REDIE) on its path towards the common manner of scientific dissemination. This research presents the evolution of Mexican policy with regards to the journals, the situation of the Educational Research Mexican journals, and its profundity relating to REDIE. In its evolution, there were three moments that stood out the most: the standardization of quality criteria, the digitalization and open access of the scientific journals, those that came together with REDIE like REDALYC and LATINDEX , and the individual initiatives of those journals that motivated the development of the scientific dissemination in Mexico. The investigation concludes with the realization of the necessity of achieving agreement on the efforts of various principal actors: governmental bodies, institutions, and academic communities.
\end{abstract}

\section{Keywords}

Electronic Journals, Education Journals, Open Access, Bibliographic Databases.

\begin{abstract}
Resumen
El propósito de este estudio es analizar el caso de la Revista Electrónica de Investigación Educativa (REDIE) en su trayecto rumbo a la corriente principal de difusión científica. Para ello, presenta la evolución de la política mexicana sobre las revistas, la situación de las revistas mexicanas de investigación educativa, y profundiza sobre la REDIE. En esta evolución se identificaron tres momentos: estandarización de los criterios de calidad, digitalización y acceso abierto de las revistas científicas, en los que confluyen instancias como REDALYC y LATINDEX y las iniciativas individuales de revistas que impulsan el desarrollo de la difusión científica del país. Se concluye sobre la necesidad de lograr la coincidencia en losesfuerzos de los diversos actores: organismos gubernamentales, instituciones y comunidades académicas.
\end{abstract}

\section{Descriptores}

Revistas Electrónicas, Revistas de Educación, acceso abierto, bases de datos bibliográficas.
Hoy en día el conocimiento científico se ha convertido en uno de los motores principales del desarrollo de las sociedades. Sin embargo, su impacto se encuentra fuertemente asociado con su potencial de diseminación. En este contexto, las revistas científicas constituyen el vehículo ideal para capitalizar dicho potencial.
Ante tal responsabilidad, las revistas científicas se convierten en blanco de constantes valoraciones y clasificaciones por parte de organismos nacionales, regionales e internacionales, los cuales, a través de sus sistemas de indización, buscan determinar la calidad, pertinencia e impacto de sus 
contenidos. En este escenario se gestan dos entornos, uno constituido por revistas cuyo contenido es considerado de alto impacto (revistas de corriente principal), y otro conformado por revistas con un impacto reducido (revistas periféricas).

El pertenecer a uno u otro entorno está directamente asociado con el logro de la revista para ser indizada y avalada por los organismos certificadores. Sin embargo, la ponderación entre cada organismo difiere, es decir, conforme las revistas avanzan hacia la indización y certificación internacional, su proximidad a la corriente principal se estrecha, y por el contrario, conforme las revistas se limitan a indizaciones nacionales o regionales se alejan. De esta forma, los sistemas de indización se han convertido en mecanismos de reconocimiento y legitimación de las revistas, y por ende de la actividad científica de los países, razón que sustenta la motivación de estas por intentar incursionar en la corriente principal de la ciencia. Este proceso de inserción de las revistas a la corriente principal exige un esfuerzo colectivo que involucre diversos actores como son organismos gubernamentales e instituciones y comunidades académicas.

Por otra parte las condiciones históricas, sociales, económicas, políticas y culturales de la ciencia latinoamericana, han propiciado que la gran mayoría de sus revistas aún sean consideradas periféricas. Ante esta realidad, surge la necesidad de que los países latinoamericanos analicen los factores que favorecen el tránsito de las revistas periféricas hacia la corriente principal de la ciencia. Pero a su vez, es importante reconocer que las condiciones particulares del contexto latinoamericano demanda que los organismos internacionales desarrollen mecanismos de evaluación de mayor pertinencia regional.

Dado lo anterior, el propósito de la presente investigación es reconstruir la evolución de los esfuerzos realizados, por el sector científico de México y sus órganos de gobierno, para habilitar la inserción de sus revistas de investigación a la corriente principal de la ciencia. A su vez, este artículo presenta las revistas mexicanas de investigación educativa, y estudio de caso a la REDIE ${ }^{[1]}$.

\section{La difusión científica a través de las revistas arbitradas}

Desde su institucionalización, la ciencia ha desarrollado un conjunto de principios, normas y mecanismos que han formalizado su dinámica, detonado su evolución y acentuado su contribución al progreso de la humanidad. Como parte de estos mecanismos, la comunicación científica se constituye como un vehículo esencial para lograr el avance del conocimiento y maximizar su impacto en la sociedad. Establecer un diálogo alrededor de los adelantos científicos no sólo detona la evolución de la ciencia, sino que la legitima. Este diálogo puede desarrollarse en dos direcciones: 1) Divulgar la ciencia, con la intención de acercar el conocimiento científico a la sociedad; y 2) Difundir la ciencia, con el propósito de distribuir el conocimiento en la comunidad científica, para que éste sea legitimado y utilizado en la construcción de nuevo conocimiento (López-Leyva, Alvarado y Mungaray, 2011).

En este contexto las revistas científicas surgen como los medios formales para difundir, organizar y evaluar la ciencia contemporánea, ya que además de dar a conocer los más recientes hallazgos científicos y validar su relevancia, pertinencia y vanguardia, también estimulan la interacción entre las comunidades académicas (Aguado-López, Rogel-Salazar, Garduño-Oropeza, Becerril-García, ZúñigaRoca y Velázquez-Álvarez, 2009; Mendoza y Paravic, 2006).

\section{a) Sistema de evaluación de las revistas}

Las revistas científicas son normadas por un conjunto de criterios e indicadores internacionales que no sólo las clasifican, sino que las legitiman y evalúan su impacto. En términos generales, estos criterios buscan garantizar la calidad de las revistas a través de la evaluación de cuatro factores: 1) Contar con una política editorial rigurosa referente al 
sistema de arbitraje, la regularidad de la publicación y la estandarización de las convenciones editoriales; 2) Cuidar la legitimidad y pertinencia del contenido de la publicación; 3) Mantener un impacto significativo, el cual se puede observar en su grado de internacionalización $\mathrm{y}$ en su visibilidad (medido en número y calidad de citas); 4) Contar con un esquema de edición y distribución que favorezca el cumplimiento de estos criterios.

Debido a que estos indicadores bibliométricos reflejan el estado de la ciencia y describen el comportamiento de la producción y consumo de la literatura académica (Cetto, 2001) son utilizados por las bases de datos bibliográficas y los sistemas de indización como criterios para determinar la inclusión o el rechazo de las revistas (Aguado-López y Rogel-Salazar, 2008).

Entre los sistemas de indización destacan dos a nivel mundial: en primer orden el Web of Science de Thomson Reuters, y auspiciado por el Institute for Scientific Information (ISI). Este sistema de indización es considerado el más influyente a nivel internacional, ya que reúne un selecto grupo de revistas con un alto grado de visibilidad y, por ende, un elevado factor de impacto, siendo éste su principal indicador bibliométrico, y el que da origen al Journal Citation Report (JCR).

Por otra parte se encuentra la base de datos Scopus, propiedad de la editorial Elsevier, la cual reúne más de 21 mil revistas científicas. Esta base de datos genera otros indicadores bibliométricos a través del SCImago Journal \& Counttry Rank (SJR). Estos indicadores no sólo estiman la relevancia de las revistas con base en el número de citas, sino que consideran otros factores -como el prestigio de las publicaciones que emiten dichas citas.

Como resultado de este complejo sistema de evaluación que otorga reconocimiento, prestigio y visibilidad global a las publicaciones científicas, emergen dos grupos de revistas, las denominadasrevistas de corriente principal y las revistas periféricas.

b) Revistas de corriente principal y revistas periféricas

A partir de la conformación y aceptación de este sistema de evaluación de las revistas, hoy en día cada una de ellas goza de diferente grado de reconocimiento y prestigio, y aquellas que se encuentran indizadas en sistemas como Web of Science (WoS) o Scopus, se convierten en el foco de interés de los académicos. Sin embargo, debido a los estrictos filtros que establecen dichos sistemas, el número de revistas que logra ingresar es reducido.

La dinámica anterior ha estimulado la conformación de una élite académica, también llamada revistas de corriente principal (Ríos y Herrero, 2005), a la que pertenecen principalmente revistas procedentes de países desarrollados, comportamiento que -como señalan Luna-Morales y Collazo-Reyes (2007)no es exclusivo de las revistas, sino de la ciencia en general.

En el caso de las revistas científicas, este predominio de los países desarrollados obedece a una amplia diversidad de factores socioeconómicos y culturales, entre ellos la adopción del idioma inglés como lingua franca de la ciencia, pero principalmente obedece al incumplimiento, por parte de las revistas, de los estándares de calidad y normalización que establecen los índices internacionales (Ríos y Herrero, 2005). No obstante, la intención de Thomson Reuters por explotar nuevos nichos de mercado, como es el caso de la región latinoamericana, lo ha llevado a definir nuevas estrategias comerciales que hoy en día favorecen el tránsito de las revistas periféricas hacia la corriente principal de la ciencia. Una de estas estrategias ha sido la alianza establecida en 2013 entre Thomson Reuters y SCIELO, para crear el Scielo Citation Index, el cual forma parte de los productos de WoS, y cuyo propósito es tener una visión crítica de la producción científica regional y su impacto en el circuito internacional. 


\section{c) Políticas latinoamericanas rumbo a la corriente principal}

Hoy en día, las revistas latinoamericanas enfrentan el reto de generar las condiciones necesarias para mejorar su situación actual, es decir, su escasa presencia en el circuito internacional y las dificultades para mantener su vigencia (Luna-Morales y Collazo-Reyes, 2007).

Sin embargo, a pesar de que gran parte de las revistas de esta región aún no logran insertarse en la corriente principal de la ciencia, hay que destacar que desde finales de los años 90 han experimentado una mejoría gradual. Tal mejora ha sido estimulada por una serie de estrategias y políticas dirigidas a acentuar la presencia de las revistas regionales dentro de la corriente principal. Estos esfuerzos se han desarrollado en varias direcciones, los primeros se orientaron a lograr una evaluación estandarizada de los criterios de calidad de las revistas, apegados siempre a los ya definidos por los índices de corriente principal.

Considerando las condiciones particulares de la región latinoamericana, se impulsaron iniciativas que resultaron en la creación de índices $\mathrm{y}$ bases de datos regionales que responden a las necesidades de su propio contexto, sin descuidar la calidad de las publicaciones. La creación de estos sistemas regionales no sólo logró homologar criterios de calidad que permitieran evaluar de manera pertinente la calidad de estas revistas, sino que potencializó su visibilidad en el contexto internacional, generando indicadores bibliométricos pertinentes a la región; y además empezaron a actuar como certificadores de calidad, difusores y orientadores de la política científica de algunos países.

Proyectos como el Sistema Regional de Información en línea para Revistas Científicas LATINDEX y la Red de Revistas Científicas REDALYC -ambos auspiciados y administrados por universidades mexicanas-, el Sistema Nacional de Indexación y Homologación de Revistas Científicas y Tecnológicas Colombianas PUBLINDEX, así como la Biblioteca virtual SCIELO -de origen brasileño y con sede en más de 10 países de Iberoamérica, se convirtieron en los motores principales de la revistas periféricas rumbo a la corriente principal de la ciencia. Aunado a lo anterior, estrategias como la alianza establecida en 2013 entre SCIELO y Thomson Reuters para desarrollar el Scielo Citation Index, se perfilan como vehículos que acelerarán la inserción de las revistas latinoamericanas en la corriente principal de la ciencia.

A manera de contraste, la tabla 1 enlista los criterios que WoS, Scopus, Scielo y Redalyc toman en cuenta para la inclusión en sus índices.

Tabla 1. Criterios de inclusión en índices internacionales

\begin{tabular}{|c|c|c|c|c|}
\hline Criterio & WoS & Scopus & Scielo & Redalyc \\
\hline Puntualidad & * & * & * & * \\
\hline Convenciones editoriales $^{a}$ & $*$ & * & * & $*$ \\
\hline Idioma $^{b}$ & $*$ & * & * & * \\
\hline Revisión por pares (RPP) & $*$ & $*$ & $*$ & $*$ \\
\hline Fuente de financiamiento & * & & & \\
\hline Diversidad internacional $^{c}$ & $*$ & $*$ & & \\
\hline Se analiza índice de autocita & & $*$ & & \\
\hline DOI & * & & & \\
\hline
\end{tabular}

a. Título de la revista, Tabla de contenidos (con títulos de los artículos), Año de publicación, Volumen y número, Resúmenes descriptivos, Número de páginas, Referencias bibliográficas completas, Adscripciones de los autores completas.

$\underline{b}$. Traducción al Inglés de: Tablas de contenido, Títulos de los artículos, Resúmenes y Descriptores o palabras clave. c. Autores y colaboradores editoriales.

Nota: Los criterios enlistados fueron tomados de las páginas de los cuatro índices que se incluyen. 
Como puede observarse, los criterios a cumplir son menos en los índices regionales, como Scielo Citation Index, que enlista 1,000 revistas en acceso abierto por ejemplo, de Ciencias, Ciencias Sociales y Artes y Humanidades de América Latina, Portugal, España, el Caribe y el sur de África; y Redalyc, que proporciona indicadores bibliométricos de 917 revistas iberoamericanas. Los criterios a cumplir son aún menos ante los organismos nacionales de cada país, como los consejos de ciencia y tecnología, aunque cada vez se acercan más a los de los índices regionales en un intento de estandarizarlos para que sus revistas puedan aspirar también a ser incluidas en los índices internacionales.

\section{Evolución de la política Mexicana sobre revistas científicas}

Países como México, Brasil y Colombia han redefinido sus agendas científicas, considerando en ellas el impulso y fortalecimiento de la difusión de la ciencia a través de las publicaciones arbitradas.

En el caso de México, el Consejo Nacional de Ciencia y Tecnología (CONACYT) ${ }^{[2]}$ ha ocupado un papel preponderante en la definición y conducción de la agenda científica nacional, $\mathrm{y}$ ha dirigido sus esfuerzos a la generación, consolidación y posicionamiento de las revistas mexicanas en la ciencia regional y mundial. Con esta visión, en 1993 el CONACYT creó el Índice de Revistas Mexicanas de Investigación Científica y Tecnológica (IRMICYT) como mecanismo de certificación de las publicaciones nacionales, a partir de ese momento se institucionalizaron los esfuerzos para estimular la difusión científica en México.

Como parte de este proceso de institucionalización se pueden distinguir tres períodos. Cada uno asociado a una estrategia específica y a un momento particular de las revistas mexicanas. El primer período inició en 1993, los esfuerzos se orientaron a lograr la formalización, estandarización y validación de las revistas. El segundo período, iniciado en el
2006, enfatizó la digitalización de las publicaciones con la finalidad de maximizar su visibilidad y optimizar los procesos editoriales y de distribución. Finalmente, en el 2014 las revistas mexicanas experimentaron una nueva etapa, en la cual se institucionalizaron los esfuerzos para impulsar el acceso abierto a las publicaciones científicas.

a) Formalización, estandarización y validación de las revistas mexicanas (1993)

A partir de la década de los 70, en México se empezaron a realizar diferentes esfuerzos para posicionar las revistas científicas en el horizonte de la ciencia internacional. Así, en 1975 y 1978 la Universidad Nacional Autónoma de México (UNAM) creó los sistemas de información bibliográfica CLASE (Citas Latinoamericanas en Ciencias Sociales y Humanidades) y PERIODICA (Índice de Revistas Latinoamericanas en Ciencias), ambos con la intención de reunir los registros bibliográficos de una amplia gama de revistas científicas latinoamericanas consideradas de calidad, sentando así las bases para la creación de otros sistemas con mayor impacto y proyección, como son los casos de LATINDEX en 1995 y REDALYC en 2002. (Haupt, 2000).

Tanto LATINDEX como REDALYC son sistemas que tienen una cobertura iberoamericana, pero que nacen bajo el impulso de instituciones mexicanas, la UNAM para el caso de LATINDEX, y la Universidad Autónoma del Estado de México (UAEM) para el caso de REDALYC.

De esta forma se infiere que durante la última década del siglo XX y los primeros años del siglo XXI, México sentó las bases de su actual sistema de evaluación de revistas científicas (López-Ornelas, 2006). Este sistema hoy en día considera cuatro criterios de calidad: 1) contenido; 2) arbitraje; 3) edición y distribución; 4) aspectos formales (CONACYT, 2013, 2012, 2010, 2009, 2008, 2006). 
En el caso del criterio contenido, el IRMICYT establece que al menos el $75 \%$ de los trabajos publicados en cada revista correspondan a artículos de investigación y que presenten resultados originales, y además debe garantizarse que el porcentaje de autores adscritos a la dependencia que edita la revista no supere el $45 \%$ en cada número publicado. Con estos indicadores el IRMICYT busca garantizar el rigor científico de las revistas, así como reducir el nivel de endogamia de las mismas (CONACYT, 2013, 2012, 2010, 2009, 2008, 2006).

En lo referente al arbitraje, el IRMICYT exige que todas las revistas implementen un estricto proceso de dictamen, para lo cual es necesario contar con una amplia cartera de árbitros constituida principalmente por evaluadores externos a la institución, y preferentemente procedentes de instituciones extranjeras. Además, los evaluadores deberán ser expertos y líderes en el área de conocimiento de la revista y gozar de amplio prestigio nacional o internacional.

Sobre el criterio de distribución, se requiere una periodicidad mínima semestral, una antigüedad mínima de tres años con una pervivencia ininterrumpida, puntualidad en la publicación y una distribución nacional e internacional.

En cuanto a los aspectos formales, el IRMICYT ha alineado sus requerimientos a los establecidos por los principales índices internacionales. Estas convenciones buscan homogeneizar la estructura y los elementos de los contenidos de las revistas, facilitando así su incorporación a sistemas internacionales de indización y certificación. Entre los elementos destacan los datos descriptivos de los artículos, como son el título, resumen, palabras clave, fechas de arbitraje, lista de dictaminadores y comité editorial, ISSN, entre otros.

\section{b) Digitalización de las revistas mexicanas} (2006)
El proceso de digitalización impulsado por el desarrollo de las TIC a partir de la segunda mitad del siglo XX, permitió que en la década de los 90 -tres siglos después de la creación de las primeras revistas científicas- surgieran las revistas electrónicas (Abadal y Rius, 2006; Laasko, Welling, Bukova, Nyman, Björk y Hedlund, 2011; Hovav y Gray, 2002). Este nuevo formato no sólo permitió hacer más eficientes los procesos editoriales, sino que impactó directamente en el proceso de distribución de las revistas. Con ello, su visibilidad creció exponencialmente, expandiendo el alcance de sus contenidos y llevándolo a un nivel global.

Sin embargo, como en cualquier proceso de adopción tecnológica, la aceptación de las revistas electrónicas por parte de los usuarios y los organismos evaluadores, implicó cierta resistencia. La carencia de rigor académico de muchas de las revistas, asociada a una etapa incipiente en la difusión y el reconocimiento de los medios digitales, fueron algunos de los factores que obstaculizaron durante, al menos una década, la consolidación y la construcción de credibilidad de las revistas electrónicas, como instrumentos formales de la difusión de la ciencia alrededor del mundo. No obstante, las comunidades científicas han sabido reconocer el potencial de las revistas electrónicas, por lo que su crecimiento dentro del entorno científico se ha intensificado durante los últimos 15 años.

En este sentido, en un esfuerzo por asegurar su credibilidad las publicaciones científicas digitales adoptaron los criterios de evaluación de las revistas científicas impresas e hicieron las adecuaciones que el entorno digital les exigía. Así, los criterios de evaluación de las revistas digitales se pueden agrupar en tres principales: aspectos formales, adecuación al medio digital y difusión e impacto (Abadal y Rius, 2006).

En México, a partir de la segunda mitad de los años 90 se emprendieron las primeras iniciativas para el desarrollo de revistas electrónicas. Una de estas iniciativas es el caso de la Revista Electrónica de Investigación 
Educativa, proyecto concebido desde su origen como una revista totalmente digital. Sin embargo, fue hasta 2006 que de acuerdo con la tendencia mundial y junto con una serie de esfuerzos por parte de diversos actores nacionales interesados en la difusión digital del conocimiento científico, como fueron las propias revistas y los sistemas de indización como REDALYC y LATINDEX, CONACYT adapta e incorpora los primeros lineamientos para evaluar las revistas electrónicas.

Un esfuerzo más por parte del CONACYT ha sido, a partir de 2014, la creación de un programa $^{[3]}$ de apoyo y asesoramiento para impulsar la migración a la plataforma Open Journal System (OJS) de todas las revistas que conforman su índice, con el objetivo de estandarizar los procesos editoriales y de gestión.

\section{c) Acceso abierto de las revistas mexicanas (2013)}

A lo largo de tres décadas, la evolución de las TIC ha situado a las revistas como elementos democratizadores de la producción y diseminación del conocimiento, marcando así un parteaguas en la comunicación de la ciencia. Desde esta perspectiva Swan (2013) señala que los componentes formales de los sistemas de edición científica se han trasladado al entorno digital y, con ello, se ha potencializado su capacidad de reproducción, visibilidad e impacto. A este contexto se suma la necesidad de reducir la asimetría en la distribución del conocimiento entre países centrales y periféricos (Aguado-López y Rogel-Salazar, 2008). Una asimetría que se ha intensificado por la orientación de los actuales sistemas de evaluación de la ciencia, particularmente por aquellos dedicados a la certificación de las revistas de corriente principal. Este escenario ha estimulado la diversificación de los esquemas de gestión, reproducción y acceso de las publicaciones científicas. Desde tal perspectiva, la influencia del acceso abierto se presenta como un elemento promotor de la distribución equitativa del conocimiento.
A través de las declaraciones de Budapest (2002), Bethesda (2003) y Berlín (2003), la comunidad promotora del acceso abierto institucionalizó sus esfuerzos para eliminar las barreras económicas, tecnológicas y regulatorias que inhiben la diseminación del conocimiento científico alrededor del mundo (Aguado-López y Rogel-Salazar; 2008). En este sentido, tres elementos se vuelven fundamentales: 1) Las revistas arbitradas que son el medio formal para difundir y evaluar la ciencia; 2) La concepción del conocimiento científico como un bien público; 3) Los medios digitales como habilitadores del libre acceso.

En su etapa inicial, el proceso de aceptación de las revistas de acceso abierto por parte de las comunidades científicas estuvo influido por una serie de cuestionamientos alrededor de su legitimidad y pervivencia. Tal y como señalan Björk y Solomon (2012), en su primera etapa estas alternativas fueron percibidas como publicaciones carentes de legitimidad, ya que al no estar certificadas por los índices internacionales, adolecían del prestigio exigido por los sistemas de evaluación de la ciencia, cuestionándose así la calidad y sostenibilidad de la revista.

No obstante, las comunidades científicas pudieron distinguir las bondades de la propuesta de acceso abierto. Las cuales Swan (2013, p. 5) sintetiza de la siguiente manera:

Mediante el Acceso Abierto, los
investigadores y estudiantes de todo el
mundo alcanzan cada vez más acceso al
conocimiento, las publicaciones obtienen
mayor visibilidad y número de lectores, y
el impacto potencial de la investigación es
fortalecido. El incremento en el acceso y
el uso compartido del conocimiento,
facilitan las oportunidades para el
desarrollo económico y social equitativo,
el diálogo intercultural, y tienen el
potencial de estimular la innovación.

En el caso de México, el fomento a la cultura del acceso abierto inicia con una serie de esfuerzos realizados por algunas Instituciones 
de Educación Superior, quienes concibieron y desarrollaron proyectos apegados a esta filosofía. Tales son los casos de la Universidad Autónoma de Baja California, con la creación de la Revista Electrónica de Investigación Educativa que nace en 1999 como una publicación de acceso gratuito y que formaliza su postura en 2004 a través de su adhesión a la iniciativa de Budapest (Cordero, LópezOrnelas, Nishikawa y McAnally, 2009), y el caso de la Universidad Autónoma del Estado de México, que desarrolla el "Sistema de Información Científica REDALYC, como uno de los proyectos regionales que procura incrementar la visibilidad de la producción científica editorial de Iberoamérica” (AguadoLópez y Rogel-Salazar, 2008, p.13).

Particularmente REDALYC se ha convertido en uno de los promotores más activos del acceso abierto en el contexto iberoamericano, $y$ en el principal promotor en el contexto nacional. De tal forma que sus esfuerzos, junto con los de CONACYT, SCIELO y otras instituciones, dieron como resultado este año la reforma a la Ley de Ciencia y Tecnología y la Ley General de Educación, estableciendo en su artículo 69 lo siguiente:

Los investigadores, tecnólogos, académicos y estudiantes de maestría, doctorado y posdoctorado, cuya actividad de investigación sea financiada con recursos públicos o que hayan utilizado infraestructura pública en su realización, por decisión personal podrán, depositar o en su caso autorizar expresamente el depósito de una copia de la versión final aceptada para publicar en Acceso Abierto a través del Repositorio Nacional, comprobando que ha cumplido con el proceso de aprobación respectivo, lo anterior bajo los términos que al efecto establezca el CONACYT (Diario Oficial de la Federación, 2014, p.1).

\section{Revistas de Investigación Educativa en México}

Las revistas de Investigación Educativa en México surgen en el segundo lustro de la década de los noventa -con algunas excepciones, como es el caso de Perfiles Educativos. De entonces a la fecha, cada una desde su trinchera y posteriormente de forma más organizada al reconocerse unas a otras, formar el Grupo de Revistas de Investigación Educativa (GRIE) y ser incluidas en el índice de revistas científicas del CONACYT, han puesto su esfuerzo en lograr el reconocimiento de organismos regionales e internacionales que avalen su calidad editorial y les den la visibilidad que tienen las revistas de corriente principal.

\section{Descripción de las revistas mexicanas dentro de los sistemas de indización}

Aunque conocidas en el ámbito nacional, ya sea porque son presentadas en congresos 0 promovidas en las páginas de diferentes universidades, la presencia de las revistas mexicanas en los índices internacionales, como el Web of Science y SCOPUS, es escasa. La cifra aumenta -aunque no de forma considerable- cuando se consultan los índices regionales, como SCIELO, REDALYC o LATINDEX, que flexibilizan sus criterios de aceptación considerando el contexto de los países en vías de desarrollo; sin embargo, contrario a lo que podría pensarse, la cifra no aumenta al revisar el índice nacional que sirve de aval ante otras instancias, situación que se da por los estrictos criterios que ha establecido, lo que incluso superan impuestos por los índices regionales. La presencia de las revistas mexicanas de educación se visualiza en la siguiente tabla. 
Tabla 2. Revistas mexicanas de educación y su presencia en índices internacionales y regionales

\begin{tabular}{|c|c|c|c|c|c|c|}
\hline & $\begin{array}{l}\text { WoS } \\
\text { Core }\end{array}$ & $\begin{array}{c}\text { Master } \\
\text { Journal } \\
\text { List } \\
\text { (WoS) }\end{array}$ & Scopus & Conacyt & Scielo & Redalyc \\
\hline RELIME & $*$ & * & * & * & * & * \\
\hline REDIE & & * & * & * & * & * \\
\hline Perfiles Educativos & & * & * & * & * & * \\
\hline RMIE & & * & * & * & * & * \\
\hline Educación Matemática & & & & * & * & * \\
\hline $\begin{array}{l}\text { Revista Iberoamericana de Educación } \\
\text { Superior }\end{array}$ & & & & & * & * \\
\hline Revista de la Educación Superior & & & & & * & * \\
\hline Revista Electrónica Sinéctica & & & & & & * \\
\hline $\begin{array}{l}\text { CPU-e, Revista de Investigación } \\
\text { Educativa }\end{array}$ & & & & & & * \\
\hline Innovación Educativa & & & & & & * \\
\hline Reencuentro & & & & & & * \\
\hline $\begin{array}{l}\text { Revista Latinoamericana de Estudios } \\
\text { Educativos }\end{array}$ & & & & & & * \\
\hline Tiempo de Educar & & & & & & * \\
\hline Apertura & & & & & & * \\
\hline
\end{tabular}

Por otro lado, en REDALYC, de las 85 revistas que se enlistan en el área de Educación,
14 revistas mexicanas cumplen con los criterios para ser indizadas.

Tabla 3. Revistas mexicanas de Educación indexadas en REDALYC

\begin{tabular}{ll}
\hline \multicolumn{1}{c}{ Revista } & \multicolumn{1}{c}{ Institución editora } \\
\hline Apertura & Universidad de Guadalajara \\
CPU-e, Revista de Investigación Educativa & Universidad Veracruzana \\
Educación Matemática & Grupo Santillana México \\
Innovación Educativa & Instituto Politécnico Nacional \\
Perfiles Educativos & Universidad Nacional Autónoma de México \\
REDIE & Universidad Autónoma de Baja California \\
Reencuentro & Universidad Autónoma Metropolitana \\
Revista de la Educación Superior & Asociación Nacional de Universidades e Instituciones de \\
& Educación Superior \\
Revista Electrónica Sinéctica & Instituto Tecnológico y de Estudios Superiores de \\
Revista Iberoamericana de Educación Superior & Occidente \\
Revista Latinoamericana de Estudios Educativos & Centro de Estudios Educativos, A.C. \\
(México) & \\
Revista Latinoamericana de Investigación en & Consejo Mexicano de Matemática Educativa \\
$\quad$ Matemática Educativa & Consejo Mexicano de Investigación Educativa, A.C. \\
Revista Mexicana de Investigación Educativa & Universidad Autónoma del Estado de México \\
\hline Tiempo de Educar
\end{tabular}

Nota: Datos tomados de http://www.redalyc.org/area.oa?id=9\&tipo=coleccion

De éstas, sólo cinco cumplen con los criterios establecidos por el CONACYT para ser incluidas en el Î́ndice de Revistas Mexicanas de Investigación Científica y Tecnológica: Revista
Latinoamericana de Investigación en Matemática Educativa (RELIME), Revista Electrónica de Investigación Educativa (REDIE), Perfiles Educativos, Revista 
Mexicana de Investigación Educativa (RMIE) y Sinéctica; las que se describen brevemente a continuación.

Perfiles Educativos. La revista Perfiles Educativos es una publicación especializada en el campo de la investigación educativa, editada por el Instituto de Investigaciones sobre la Universidad y la Educación (IISUE) de la Universidad Nacional Autónoma de México. Su finalidad es difundir los resultados de la investigación educativa y fomentar el debate académico en el ámbito iberoamericano.

REDIE. La REDIE es una publicación cuatrimestral especializada en investigación educativa, editada por el Instituto de Investigación y Desarrollo Educativo (IIDE) de la Universidad Autónoma de Baja California. Su objetivo es difundir y promover la investigación en educación en el ámbito nacional y latinoamericano.

RELIME. La RELIME es una publicación cuatrimestral editada por el Colegio Mexicano de Matemática Educativa a través del Comité Latinoamericano de Matemática Educativa. Nace como un foro para las diversas escuelas del pensamiento (paradigmas, teorías, métodos) respetando las tradiciones educativas y contextos de los sistemas educativos de la región.

RMIE. La RMIE se edita desde 1996 por el Consejo Mexicano de Investigación Educativa. Publica resultados de investigación realizados en México y Latinoamérica con el propósito de contribuir al fortalecimiento de la investigación de calidad en la región. Desde junio de 2002 cuenta con una versión electrónica.

Sinéctica. Sinéctica, Revista Electrónica de Educación, es editada por el Iteso, Universidad Jesuita de Guadalajara. Aborda la problemática social desde el campo educativo y promueve la producción académica de calidad en ese campo. Publica artículos con temáticas variadas sobre el campo educativo y en cada número incluye una sección monotemática.

\section{Estandarización, digitalización y acceso abierto: el caso de la REDIE}

A continuación se presenta una descripción de las acciones emprendidas por la REDIE desde su concepción hasta la actualidad. Esta descripción toma como ejes de discusión los tres factores que han transformado la dinámica de la difusión científica mundial, es decir, la estandarización de los criterios de calidad de las revistas, su digitalización y su postura ante el acceso abierto. Factores que, a su vez, se constituyen en estrategias que habilitan el tránsito de las revistas periféricas hacia la corriente principal de la ciencia.

\section{La REDIE ante la estandarización de los criterios de calidad}

Con el primer número publicado en 1999, la Revista Electrónica de Investigación Educativa iniciaba su camino como una publicación sui géneris en México y Latinoamérica, debido a sus características editoriales: electrónica, arbitrada y de acceso abierto. Sin embargo, el hecho de tener estas características implicó un esfuerzo extra por parte del equipo editorial para desarrollar y consolidar un proyecto que, por novedoso en el contexto regional, no inspiraba la confianza que una revista académica impresa daba tanto a autores $\mathrm{y}$ editores de otras revistas, como a las instancias evaluadoras a nivel nacional-mexicano-, en este caso al CONACYT.

Aunque en el momento de publicación de la REDIE las revistas electrónicas a nivel internacional eran reconocidas ya como medios de difusión del conocimiento científico (Área, 1998), la REDIE tuvo que hacer frente al poco conocimiento que se tenía y a la desconfianza de muchos investigadores, pues se desconocía el funcionamiento y los procesos para publicar en un medio electrónico, además de la incertidumbre que había sobre la "permanencia” de la revista, pues al no estar impresa se percibía como algo intangible.

Lograr el reconocimiento y aceptación de la revista fue un reto: ser una publicación 
electrónica fue el primero y más grande, pues en ese momento las pocas publicaciones de este tipo en México no contaban con lo que ahora denominamos criterios editoriales (ISSN, cartera de árbitros, normas editoriales, etc.), lo que hacía que se desconfiara de ellas; por ese motivo, desde su creación, la REDIE se dio a la tarea de contar con los criterios que la definieran como una revista académica formal, y para ello -además de considerar los criterios básicos internacionales- buscó cubrir los requisitos que el Índice de Revistas Mexicanas de Investigación Científica y Tecnológica pedía a las revistas impresas, que hasta ese momento no consideraba a las revistas electrónicas.

El segundo reto fue obtener la reserva de derechos ante el Instituto Nacional del Derecho de Autor (Indautor), que para otorgar el registro exigió la presentación impresa del primer número de la revista, pues registrar una revista electrónica era algo inédito hasta ese momento. Mientras que, por otro lado, el ISSN tuvo que realizarse en la oficina de París, ya que la oficina en México no reconocía como válida la publicación electrónica.

El tercer reto, y el más arduo quizá, fue obtener la confianza de autores y revisores, pues una revista nueva, con características aún más novedosas, despertaba una natural desconfianza.

Cumplir con los criterios de normalización de una publicación académica le permitió a la REDIE proponer la inclusión de las revistas electrónicas en el índice de CONACYT y abrirse así a nuevas plataformas de difusión del conocimiento científico (Cordero, LópezOrnelas, Nishikawa y McAnally, 2009), esto dio como resultado que en 2002 la REDIE se convirtiera en la primera publicación electrónica en ingresar al índice más importante del país; con su inclusión, y a partir de los criterios que la regían, la REDIE abrió las puertas para que las revistas electrónicas adquirieran legitimidad ante la comunidad académica y las instancias evaluadoras.

A 15 años de distancia, la REDIE se ha ajustado a los criterios de los principales índices internacionales, y ha estandarizado el formato y el estilo de los artículos que publica cuatrimestralmente (desde el 2013) en html y PDF. El apego a los criterios de calidad y el hecho de ser objeto de investigación del equipo que trabaja en ella, ha permitido obtener datos sobre el alcance de la publicación; esta búsqueda de mejora constante ha hecho que la REDIE permanezca y se consolide como un medio de difusión confiable.

\section{La REDIE ante la digitalización}

Frente a la necesidad e interés de las comunidades académicas por potenciar la diseminación de sus contribuciones, y frente a los atributos desarrollados por las TIC como parte de su proceso evolutivo, surgen las revistas electrónicas en dos modalidades: 1) Las revistas impresas que decidieron complementar su proceso de distribución con una versión electrónica, y que naturalmente responden a un modelo económico basado en la suscripción; y 2) Las revistas que nacen totalmente electrónicas, y que tienen un mayor potencial para adoptar un modelo de acceso abierto.

En este contexto la REDIE se concibió como la primera revista mexicana de investigación educativa totalmente electrónica y de acceso abierto a texto completo. Esta decisión no sólo contribuyó a incrementar exponencialmente la visibilidad de sus contenidos, sino que añadió otras ventajas como la reducción de costos de producción y distribución, el enriquecimiento de los documentos a partir de la diversidad de recursos y formatos, así como la internacionalización de la revista a través de la participación de autores, lectores y árbitros procedentes de todo el mundo.

Es importante mencionar que aunque la REDIE es una revista que nace digital, esta ha experimentado una evolución en torno a su proceso de digitalización y sistematización, el cual se condujo en dos sentidos: 1) Digitalizar su distribución y forma de acceso; y 2) Sistematizar su proceso de gestión editorial. Respecto al primer punto, la REDIE en sus inicios (1999-2004) fue desarrollada con base en un sistema de páginas estáticas, que 
posteriormente evolucionó hacia el desarrollo de una plataforma de código abierto y páginas dinámicas, lo que evitó el pago de licencias y facilitó la adaptación de la plataforma a los requerimientos de la revista. Mientras que en lo referente a la sistematización del proceso de gestión editorial, este se llevó a cabo durante el 2013, momento en el que la REDIE migró a la plataforma Open Journal System (OJS), a fin de hacer más eficiente la presentación y el flujo editorial de la revista.

A pesar de las grandes ventajas que la digitalización ofrece a la difusión científica, en sus inicios la REDIE experimentó una serie de retos asociados con la cultura, la adopción y la alfabetización tecnológica por parte de los lectores. Inquietudes sobre la integridad, permanencia y legitimidad de los contenidos, así como sobre el uso del medio digital, fueron argumentos recurrentes $\mathrm{y}$ por momentos condicionaron su aceptación, no sólo por parte de los usuarios y autores, sino por los propios organismos evaluadores.

En este sentido, la REDIE adoptó una postura proactiva, definiendo lineamientos y criterios para la evaluación pertinente de las revistas electrónicas. Esos lineamientos se presentaron en 2002 y 2005 ante CONACYT, junto con una solicitud para que las revistas electrónicas fueran consideradas por el IRMICYT, siendo estos lineamientos incluidos por primera vez en la Convocatoria 2006 de ingreso $y$ permanencia de revistas científicas al IRMICYT (López-Ornelas, 2006). Desde esta perspectiva se puede observar que la REDIE, a lo largo de su historia, ha mantenido un interés permanente por contribuir de manera activa e impulsar la evolución de los mecanismos institucionales y la política nacional sobre difusión de la ciencia.

\section{La REDIE ante el acceso abierto}

Hasta antes de la digitalización de las revistas científicas, la difusión de la ciencia dependía de un paradigma tecno-económico que permitía que las editoriales, a través de una cesión de derechos por parte de los autores, restringieran el acceso a los contenidos científicos mediante suscripciones, licencias o pago por artículo. Este paradigma, que operó por más de tres siglos, atentaba contra una de las funciones principales de la ciencia, la difusión del conocimiento (McAnally, 2005). Sin embargo, con la llegada de las TIC y su amplia gama de capacidades para producir y distribuir información, se redujo la necesidad de recurrir a este tipo de intermediarios.

Ante el interés de universidades y académicos por preservar y dar a conocer sus contribuciones al avance de la ciencia, el acceso abierto emerge como una opción que le otorga a los productores de conocimiento la capacidad de decidir sobre la restricción o el libre acceso a sus contenidos.

Es en este contexto en el que la REDIE, con el apoyo de la institución que la auspicia (Universidad Autónoma de Baja California), nace como una revista arbitrada de acceso libre a texto completo. La razón principal detrás de esta decisión radica en la motivación original de la creación de la revista y en la responsabilidad asumida por la propia universidad por difundir el conocimiento. En esta línea de pensamiento Cordero et al. (2009, p.6) señalan:

"El nacimiento de la REDIE como una revista de acceso gratuito a sus contenidos fue novedoso en la institución donde se edita, y generó una situación de reflexión al interior del equipo editorial de la REDIE. Pensar en el papel de la universidad en la difusión del conocimiento, sobre todo como institución pública, motivó a tomar una posición mucho más explícita en torno al acceso abierto. Con el tiempo, surgió la necesidad de dejar claro que esta postura no era un elemento circunstancial en la Revista, sino un posicionamiento voluntario, producto de la reflexión, y una verdadera convicción. Por ello, a partir de noviembre de 2004 la REDIE se adhirió a la Iniciativa de Budapest sobre Acceso Abierto”. 
No obstante, la concepción de la REDIE como una revista de acceso abierto no se limitó al simple acceso a sus contenidos, sino también a su uso y distribución sin restricciones por parte de los lectores. Por ello, se decidió definir de manera explícita una política en torno al derecho de autor, e instrumentar los mecanismos legales necesarios para sustentar dicha política. Es así como se decidió adoptar la licencia Atribución- No comercial 2.5 México, de Creative Commons, la cual garantiza el libre uso de los contenidos, respetando tres elementos fundamentales: el reconocimiento del derecho moral sobre la obra, la utilización sin fines de lucro y mantener la integridad del contenido (Cordero et al. 2009).

Asumir el riesgo de crear una revista totalmente gratuita no fue una decisión fácil, ya que no sólo implicaba la incertidumbre sobre la sostenibilidad financiera de la misma, implicaba además abandonar un esquema cultural y organizativo añejo, errónea y frecuentemente asociado con la credibilidad y la seriedad de la revista (McAnally, 2005). Sin embargo, la misión de la revista de contribuir a la difusión del conocimiento científico actuó como el factor determinante de esta postura.

Congruente con esta línea de pensamiento, en el año 2014 a exactamente 15 años del nacimiento de la REDIE como una publicación electrónica y de acceso abierto, CONACYT ha definido e institucionalizado una postura nacional sobre el acceso abierto a los resultados de investigación financiados con recursos públicos, a través de la reforma del artículo 69 de la ley de ciencia y tecnología, hecho que reafirma la pertinencia de las posturas asumidas por la REDIE a lo largo de su trayectoria.

\section{Conclusiones}

La presente investigación se enfocó en identificar los elementos o estrategias que habilitan la transición de las revistas periféricas hacia la corriente principal de la ciencia, esto a partir de la reconstrucción de los esfuerzos realizados por el sector científico de México, y analizando el caso de la REDIE.
En este escenario destaca la preponderancia de las revistas científicas como difusoras del conocimiento, así como la necesidad de su certificación por parte de los sistemas de indización, ya que estos actúan como mecanismos de legitimación de la producción científica publicada. A su vez, se establece la diferencia entre la corriente principal de la ciencia y la corriente periférica, siendo esta última a la que pertenece la mayoría de revistas latinoamericanas, las cuales ven limitado su impacto, entre otros factores, por el idioma y dificultades en la gestión editorial, donde la digitalización y el acceso abierto son los medios que potencializan la visibilidad.

Por otra parte, el análisis del comportamiento de las revistas mexicanas de investigación educativa ante los sistemas de indización nacionales, regionales e internacionales, evidencia que los sistemas con menor rigor durante la evaluación de las revistas son los nacionales y regionales, como es el caso de REDALYC, SCIELO y el IRMICYT, los cuales presentan mayor flexibilidad en los criterios de calidad, lo que demuestra que estos sistemas han sido pensados para atender las condiciones del contexto latinoamericano. Mientras que los sistemas internacionales como SCOPUS $y$ Web of Science mantienen un esquema de evaluación más riguroso, privilegiando los factores de citación e impacto, criterios que suelen favorecer a las revistas de habla inglesa y procedentes de los países desarrollados. Prueba de ello es el comportamiento que registra cada revista seleccionada para este estudio, en donde se hace evidente cómo disminuye la presencia de dichas revistas en índices internacionales, en comparación con los regionales.

A su vez, se observó que el tránsito de las revistas -de la zona periférica a la corriente principal- es un esfuerzo colectivo de orden nacional que involucra diferentes actores, empezando por el nivel gubernamental, pasando por el nivel institucional y terminando con el nivel individual, es decir, los propios académicos y sus comunidades. 
En el caso de México, la institucionalización de estos esfuerzos empezó a partir de iniciativas llevadas a cabo por comunidades e instituciones académicas, que a través de la creación de revistas y sistemas de indización fueron impulsando la gestación de una política nacional de revistas científicas, en torno a tres elementos que han revolucionado la dinámica de la difusión científica en el mundo, y que actualmente pueden visualizarse como estrategias para incursionar en la corriente principal. Estas estrategias son: 1) $L a$ estandarización de los criterios de calidad de las revistas; 2) La digitalización de las revistas; y 3) El acceso abierto a las revistas.

Si bien es cierto que el seguimiento de estas tres estrategias no garantiza la transición de las revistas periféricas a la corriente principal, sí afianza los pasos hacia su acercamiento, ya que potencializan su visibilidad y facilitan su gestión editorial, favoreciendo el cumplimiento de la mayoría de los criterios establecidos por los sistemas internacionales. Prueba de ello es el caso de la REDIE, publicación que en el lapso de 15 años de vida, ha avanzado en su trayecto hacia la corriente principal, siendo indizada por los principales sistemas nacionales, regionales e internacionales, por ejemplo: IRMICYT, REDALYC, SICELO, SCOPUS, y Web of Science, y que hoy la ubican en la antesala del núcleo de la corriente principal de la ciencia. Sin duda, el éxito de la REDIE parte de su concepción y de su compromiso con la difusión del conocimiento científico, no sólo en el país, sino en el mundo.

De esta forma, el principal reto de las revistas mexicanas de investigación educativa es alcanzar y mantener la excelencia científica de sus contenidos, y desarrollar su capacidad para intensificar su visibilidad e impacto en el circuito internacional de la ciencia.

\section{Referencias}

Abadal, E. y Rius, Ll. (2006). Revistas científicas digitales: características e indicadores. Revista de Universidad $y$ Sociedad del Conocimiento, 3(1), 6-20.
Recuperado

de http://www.uoc.edu/rusc/3/1/dt/esp/abadal rius. pdf

Aguado-López, E., Rogel-Salazar, R., GarduñoOropeza, G., Becerril-García, A., ZúñigaRoca, M. F. y Velázquez-Álvarez, A. (2009). Patrones de colaboración científica a partir de redes de coautoría. Convergencia. Revista de Ciencias Sociales, Núm. Esp. IA, 225-258.

Aguado-López, E. y Rogel-Salazar (2008). Redalyc: una alternativa a las asimetrías en la distribución del conocimiento científico. Ciencia, Docencia y Tecnología, 31(19), 11-30.

Area, M. (1998). ¿Y a partir de ahora qué? Del aislamiento a la colaboración entre los docentes e investigadores españoles en tecnología educativa. Trabajo presentado en las VI Jornadas Universitarias de Tecnología Educativa, Tenerife, España.

Björk, B. y Solomon, D. (2012). Open access versus subscription journals: a comparison of scientific impact. MBC Medicine, 10(73). Recuperado de http://www.biomedcentral.com/1741$\underline{7015 / 10 / 73}$

Consejo Nacional de Ciencia y Tecnología (CONACYT). (2013). Criterios generales de evaluación para el índice de revistas mexicanas de investigación científica y tecnológica del CONACYT, 2013. Autor. Recuperado de

http://www.conacyt.mx/index.php/elconacyt/convocatorias-y-resultadosconacyt/convocatorias-indice-revistas-cyt1/convocatorias-cerradas-indice-revistasinvestigacion-1/convocatorias-cerradas2013-indice-revistas-1/2528--251/file

CONACYT (2012). Criterios generales de evaluación para el índice de revistas mexicanas de investigación científica y tecnológica del CONACYT, 2012. Autor. Recuperado de http://www.conacyt.mx/index.php/elconacyt/convocatorias-y-resultadosconacyt/convocatorias-indice-revistas-cyt- 
$\underline{1 / \text { convocatorias-cerradas-indice-revistas- }}$ investigacion-1/convocatorias-cerradas2012-indice-revistas-1/2535--257/file

CONACYT. (2010). Criterios generales de evaluación para el índice de revistas mexicanas de investigación científica $\mathrm{y}$ tecnológica del conacyt, 2010-2011.

Recuperado de

http://www.conacyt.mx/index.php/elconacyt/convocatorias-y-resultadosconacyt/convocatorias-indice-revistas-cyt1/convocatorias-cerradas-indice-revistasinvestigacion-1/convocatorias-cerradas-20102011-indice-revistas-1/2540--260/file

CONACYT. (2009). Criterios generales de evaluación para el índice de revistas mexicanas de investigación científica y tecnológica del CONACYT, 2009. Recuperado de

http://www.conacyt.mx/index.php/elconacyt/convocatorias-y-resultadosconacyt/convocatorias-indice-revistas-cyt$1 /$ convocatorias-cerradas-indice-revistasinvestigacion-1/convocatorias-cerradas2009-indice-revistas-1/2545--264/file

CONACYT. (2008). Criterios generales de evaluación para el índice de revistas mexicanas de investigación científica y tecnológica del CONACYT, 2008. Recuperado de

http://www.conacyt.mx/index.php/el-

conacyt/convocatorias-y-resultados-

conacyt/convocatorias-indice-revistas-cyt-

$\underline{1 / \text { convocatorias-cerradas-indice-revistas- }}$ investigacion-1/convocatorias-cerradas2008-indice-revistas-1/2549--266/file

CONACYT. (2006). Criterios generales de evaluación para el índice de revistas mexicanas de investigación científica y tecnológica del CONACYT, 2006-2007. Recuperado de http://www.conacyt.mx/index.php/elconacyt/convocatorias-y-resultadosconacyt/convocatorias-indice-revistas-cyt$\underline{1 / \text { convocatorias-cerradas-indice-revistas- }}$ investigacion-1/convocatorias-cerradas2006-2007-indice-revistas-1/2553--268/file
Cordero, G., López-Ornelas, M., Nishikawa, K. A. y McAnally, L. (2009). Diez años de vida en línea: la experiencia de editar una revista electrónica en educación. Revista Electrónica de Investigación Educativa, 11(2), 1-24. Recuperado de http://redie.uabc.mx/index.php/redie/article/ view/238/755

Cetto, A. M. (2001). ¿Qué futuro tienen las revistas latinoamericanas?. Ciencia y Mar, 13, 37-42. Recuperado de http://www.umar.mx/revistas/13/amc.pdf

Diario Oficial de la Federación. (2014). Decreto por el que se reforman y adicionan diversas disposiciones de la Ley de Ciencia y Tecnología, de la Ley General de Educación y de la Ley Orgánica del Consejo Nacional de Ciencia y Tecnología. Publicado el 20 de mayo de 2014. Recuperado de http://www.dof.gob.mx/nota_detalle.php?co $\underline{\text { digo }}=5345503 \&$ fecha $=20 / 05 / 2014$

Haupt, C. (2000). Las revistas científicas latinoamericanas: su difusión y acceso a través de base de datos. Biblioteca Universitaria (nueva época), 3(2), 122-127. Recuperado de http://dgb.unam.mx/servicios/dgb/publicdgb bole/fulltext/volIII2/octava.PDF

Hovav, A. y Gray, P. (2002). Future penetration of academic electronic journals: Four scenarios. Information Systems Frontiers, 4(2), 229-244. Recuperado de http://148.231.10.114:2804/static/pdf/137/ar t\%253A10.1023\%252FA\%253A101605900 4916.pdf?auth66=1414114369_90b88aa19c 42b779fd20558f0d20da0d\&ext $=. p d f$

Laakso, M., Welling, P., Bukvova, H., Nyman, L., Björk, B-C y Hedlund, T. (2011). The development of open access journal publishing from 1993 to 2009. PLoS ONE, doi: 10.1371/journal.pone.0020961.

López-Leyva, S., Alvarado-Borrego, A. y Mungaray-Moctezuma, A. B. (2011). La comunicación de la ciencia a través de artículos científicos. México: Juan Pablos Editores y Universidad de Occidente. 
Luna-Morales, M. E. y Collazo-Reyes, F. (2007). Análisis histórico bibliométrico de las revistas latinoamericanas y caribeñas en los índices de la ciencia internacional: 1961-2005. Revista Española de Documentación Científica, 30(4), 523-543.

Mendoza, S. y Paravic, T. (2006). Origen, clasificación y desafíos de las revistas científicas. Investigación y Postgrado, 21(1), 49-76.
Ríos, C. y Herrero, V. (2005). La producción científica latinoamericana y la ciencia mundial: una revisión bibliográfica (19892003). Revista Interamericana de Bibliotecología, 28(1), 43-61.

Swan, A. (2013). Directrices para políticas de desarrollo y promoción del acceso abierto. Francia: Organización de las Naciones Unidas para la Educación, la Ciencia y la Cultura.

\section{NOTAS}

${ }^{[1]}$ La REDIE es la primera revista mexicana que se concibe en un formato digital y de acceso abierto. Aunque nació en el contexto de las revistas periféricas, ha enfocado sus esfuerzos en disminuir la distancia que la separa de las revistas de corriente principal.

[2] CONACYT es un organismo gubernamental mexicano que impulsa y fortalece el desarrollo científico, a través de la definición de políticas públicas y de programas de estímulo a la ciencia y a la tecnología.

[3] Liga al Programa Guiado para la Adopción de la plataforma OJS: http://conacyt.conocimientovirtual.org 


\section{ABOUT THE AUTHORS / SOBRE LOS AUTORES}

Cabrera-Flores, Mayer (mayer.cabrera@uabc.edu.mx). Doctor en Estudios del Desarrollo Global por la Universidad Autónoma de Baja California (UABC). Es académico del Instituto de Investigación y Desarrollo Educativo de la UABC, Editor de diagramación de la Revista Electrónica de Investigación Educativa (REDIE), y colaborador del cuerpo académico de Tecnología Digital en Educación. Sus líneas de investigación son Ecosistemas de Innovación; Política Científica, Tecnológica y de Educación Superior; Difusión y Divulgación de la Ciencia; Educación y Cambio Tecnológico. Su dirección postal es: Instituto de Investigación y Desarrollo Educativo. Universidad Autónoma de Baja California. Km. 103 Carretera Tijuana-Ensenada. C.P. 22860 Ensenada, Baja California, (México). Buscar otros artículos de esta autora en Google Académico / Find other articles by this author in Scholar Google Google

Luna-Serrano, Edna (eluna@uabc.edu.mx). Investigadora del Instituto de Investigación y Desarrollo Educativo, Universidad Autónoma de Baja California (Baja California, México). Es la autora de contacto para este artículo. Coordinadora del Doctorado en Ciencias Educativas de la UABC. Responsable del Cuerpo Académico “Evaluación Educativa” nivel consolidado. Es directora de REDIE (Revista electrónica de investigación educativa). Su dirección postal es: Instituto de Investigación y Desarrollo Educativo. Universidad Autónoma de Baja California. Km. 103 Carretera Tijuana-Ensenada. C.P. 22860 Ensenada, Baja California, (México). Buscar otros artículos de esta autora en Google Académico/Find other articles by this author in Scholar Google

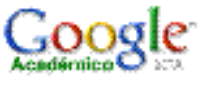

Vidauri, Gabriela (gvidauri@uabc.edu.mx). Especialista en Lengua y Literatura de Hispanoamérica y estudiante de la maestría en Cultura Escrita. Actualmente es Editora de Producción de laRevista Electrónica de Investigación Educativa (REDIE) y técnico académico de investigación asociada al cuerpo académico de Discurso, Identidad y Prácticas Educativas en el Instituto de Investigación y Desarrollo Educativo de la Universidad Autónoma de Baja California. Su dirección postal es: Instituto de Investigación y Desarrollo Educativo. Universidad Autónoma de Baja California. Km. 103 Carretera Tijuana-Ensenada. C.P. 22860 Ensenada, Baja California, (México). Buscar otros artículos de esta autora en Google Académico / Find other articles by this author in Scholar Google 


\title{
ARTICLE RECORD / FICHA DEL ARTÍCULO
}

\begin{tabular}{|c|c|}
\hline $\begin{array}{l}\text { Reference / } \\
\text { Referencia }\end{array}$ & 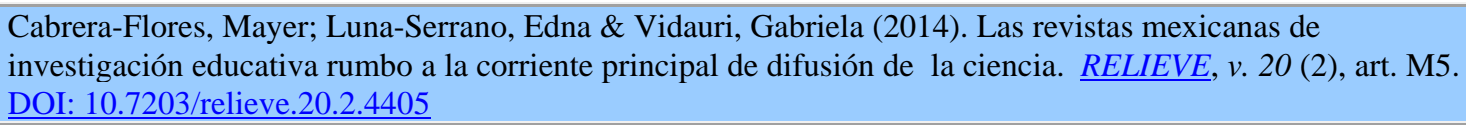 \\
\hline Title / Título & $\begin{array}{l}\text { Las revistas mexicanas de investigación educativa rumbo a la corriente principal de difusión de la } \\
\text { ciencia. [Mexican journals of educational research towards mainstream dissemination of science]. }\end{array}$ \\
\hline $\begin{array}{l}\text { Authors / } \\
\text { Autores }\end{array}$ & Cabrera-Flores, Mayer; Luna-Serrano, Edna \& Vidauri, Gabriela \\
\hline Review / Revista & RELIEVE (Revista ELectrónica de Investigación y EValuación Educativa), v. 20 n. 2 \\
\hline ISSN & $1134-4032$ \\
\hline $\begin{array}{l}\text { Publication date / } \\
\text { Fecha de } \\
\text { publicación }\end{array}$ & $\begin{array}{l}2014 \text { (Reception Date: } 2014 \text { October } 22 \text {; Approval Date: } 2014 \text { December 2. Publication Date: } 2014 \\
\text { December 05) }\end{array}$ \\
\hline \multirow{2}{*}{$\begin{array}{l}\text { Abstract / } \\
\text { Resumen }\end{array}$} & $\begin{array}{l}\text { The purpose of this study is to analyze the Electronic Journal of Educational Research (REDIE) on its path } \\
\text { towards the common manner of scientific dissemination. This research presents the evolution of Mexican } \\
\text { policy with regards to the journals, the situation of the Educational Research Mexican journals, and its } \\
\text { profundity relating to REDIE. In its evolution, there were three moments that stood out the most: the } \\
\text { standardization of quality criteria, the digitalization and open access of the scientific journals, those that } \\
\text { came together with REDIE like REDALYC and LATINDEX, and the individual initiatives of those journals } \\
\text { that motivated the development of the scientific dissemination in Mexico. The investigation concludes with the } \\
\text { realization of the necessity of achieving agreement on the efforts of various principal actors: governmental } \\
\text { bodies, institutions, and academic communities. }\end{array}$ \\
\hline & $\begin{array}{l}\text { El propósito de este estudio es analizar el caso de la Revista Electrónica de Investigación Educativa } \\
\text { (REDIE) en su trayecto rumbo a la corriente principal de difusión científica. Para ello, presenta la evolución } \\
\text { de la política mexicana sobre las revistas, la situación de las revistas mexicanas de investigación educativa, y } \\
\text { profundiza sobre la REDIE. En esta evolución se identificaron tres momentos: estandarización de los criterios } \\
\text { de calidad, digitalización y acceso abierto de las revistas científicas, en los que confluyen instancias como } \\
\text { REDALYC y LATINDEX y las iniciativas individuales de revistas que impulsan el desarrollo de la difusión } \\
\text { científica del país. Se concluye sobre la necesidad de lograr la coincidencia en los esfuerzos de los diversos } \\
\text { actores: organismos gubernamentales, instituciones y comunidades académicas. }\end{array}$ \\
\hline $\begin{array}{l}\text { Keywords / } \\
\text { Descriptores }\end{array}$ & $\begin{array}{l}\text { Electronic Journals, Education Journals, Open Access, Bibliographic Databases. } \\
\text { Revistas Electrónicas, Revistas de Educación, acceso abierto, bases de datos bibliográficas }\end{array}$ \\
\hline $\begin{array}{l}\text { Institution / } \\
\text { Institución }\end{array}$ & Universidad Autónoma de Baja California (México) \\
\hline $\begin{array}{l}\text { Publication site / } \\
\text { Dirección }\end{array}$ & http://www.uv.es/RELIEVE \\
\hline $\begin{array}{l}\text { Language / } \\
\text { Idioma }\end{array}$ & Español \& English version (Title, abstract and keywords in English \& Spanish) \\
\hline
\end{tabular}

\section{RELIEVE}

\section{Revista ELectrónica de Investigación y $\mathbf{E V}$ aluación $\mathbf{E d u c a t i v a ~}$ E-Journal of Educational Research, Assessment and Evaluation}

\author{
[ISSN: 1134-4032]
}

\begin{abstract}
(C) Copyright, RELIEVE. Reproduction and distribution of this articles it is authorized if the content is no modified and their origin is indicated (RELIEVE Journal, volume, number and electronic address of the document).

(C) Copyright, RELIEVE. Se autoriza la reproducción y distribución de este artículo siempre que no se modifique el contenido y se indique su origen (RELIEVE, volumen, número y dirección electrónica del documento).
\end{abstract}

